\title{
Sobolev Mappings with Integrable Dilatations
}

\author{
Juha Heinonen \& PekKa Koskela
}

Communicated by J. M. BALL

\begin{abstract}
We show that each quasi-light mapping $f$ in the Sobolev space $W^{1, n}\left(\Omega, \mathbf{R}^{n}\right)$ satisfying $|D f(x)|^{n} \leqq K(x, f) J(x, f)$ for almost every $x$ and for some $K \in L^{r}(\Omega), r>n-1$, is open and discrete. The assumption that $f$ be quasilight can be dropped if, in addition, it is required that $f \in W^{1, p}\left(\Omega, \mathbf{R}^{n}\right)$ for some $p \geqq n+1 /(n-2)$. More generally, we consider mappings in the John Ball classes $\mathscr{A}_{p, q}(\Omega)$, and give conditions that guarantee their discreteness and openness.
\end{abstract}

\section{Introduction}

Let $f$ be a mapping in the Sobolev space $W^{1, p}\left(\Omega, \mathbf{R}^{n}\right)$, where $\Omega \subset \mathbf{R}^{n}$ is open, $n \geqq 2$, and $1 \leqq p \leqq \infty$. A fundamental problem in nonlinear elasticity is to determine what analytic data on $f$ provide topological information on the deformation that $f$ represents. For instance, under what conditions is $f$ locally or globally invertible? Does $f$ map open sets to open sets? These problems have been studied in a series of articles by BALL and others; see [B1, B2, S, I $\check{S}$, MTY] and the references therein. The purpose of this paper is to give some further results in this direction. Before we formulate our objective more precisely, we introduce some terminology.

Throughout this paper $|D f(x)|$ designates the supremum norm of the differential $D f(x)$ with $J(x, f)=\operatorname{det} D f(x)$ its Jacobian determinant; they exist for almost every $x$ in $\Omega$.

Definition. A measurable function $K(\cdot, f)$ in $\Omega$ is called a dilatation of $f$ if

$$
\begin{gathered}
1 \leqq K(x, f)<\infty \quad \text { for almost every } x \in \Omega, \\
|D f(x)|^{n} \leqq K(x, f) J(x, f) \quad \text { for almost every } x \in \Omega .
\end{gathered}
$$

We say that $f$ is a mapping of finite dilatation if a function $K(\cdot, f)$ exists satisfying (1.1) and (1.2). 
The above terminology is essentially the same as used by IwanIEC $\&$ ŠVERÁK [IŠ] . They assume that $J(x, f) \geqq 0$ a.e. and that $D f(x)=0$ whenever $J(x, f)=0$; they then define $K(x, f)$ to be the quotient $|D f(x)|^{n} / J(x, f)$, whenever this exists, and $K(x, f)=1$ otherwise. The usual regularity assumption on $f$ in this connection would be $f \in W^{1, n}\left(\Omega, \mathbf{R}^{n}\right)$, which trivially gurantees the integrability of the Jacobian $J(x, f)$. However, recent studies have shown that there are natural, weaker integrability hypotheses on $D f$ guaranteeing the same conclusion; see, e.g., [IS, MTY]. Notice that for a mapping of finite dilatation we have $J(x, f) \geqq 0$ a.e. and that any mapping satisfying $J(x, f)>0$ a.e. is a mapping of finite dilatation.

The following theorem lists some important properties of mappings of finite dilatation in $W^{1, n}\left(\Omega, \mathbf{R}^{n}\right)$ :

1.3. Theorem. Let $f \in W^{1, n}\left(\Omega, \mathbf{R}^{n}\right)$ be a mapping of finite dilatation. Then

(1) $f$ has a continuous representative (also called $f$ ),

(2) locally

$$
|f(x)-f(y)| \leqq C(n)\|D f\|_{n}\left(\log \frac{2}{|x-y|}\right)^{-1 / n},
$$

(3) $f$ is differentiable a.e.,

(4) $f$ is sense-preserving,

(5) $f$ satisfies condition $(N)$, i.e., $|f(E)|=0$ whenever $E \subset \Omega$ is such that $|E|=0$, where $|\cdot|$ stands for Lebesgue n-measure.

We recall that a continuous mapping $f: \Omega \rightarrow \mathbf{R}^{n}$ is sense-preserving if the topological index $\mu(y, f, D)$ is positive for all domains $D \subset \subset \Omega$ and $y \in f(D) \backslash f(\partial D)$. We refer to [R, p. 85] or [Ri, Chapter 1] for the precise definition and basic properties of the topological index.

The adverb "locally" in (2) can be made more precise: for each compact $F \subset \Omega$ there is a constant $\delta>0$, depending only on $\operatorname{dist}(F, \partial \Omega)$, such that (1.4) holds for each pair of points $x, y \in F$ with $|x-y|<\delta$.

Proof. Assertion (1) is due to Gol'DShTEIN \& Vodop'yanov [GV]; they show that $f$ is monotone, and the proof in [R, p. 339] can be modified so as to yield the continuity together with (2) (and the more precise statement cited after the theorem). A more elementary proof of (1) was recently given by MANFREDI [M]. See also [Š].

Now (3) follows from the proof of Theorem 1.2 in [R, p. 84]; the only property of quasiregular mappings used in that proof is the equicontinuity of a family of maps, but this property is guaranteed by (2).

Next, by [Ri, Lemma 5.1] $f$ is weakly sense-preserving, and by the proof of [Ri, I.4.5] $f$ is actually sense-preserving (see also [R, p. 175]). The crucial point is that for mappings of finite dilatation the differential $D f(x)$ vanishes at almost every point $x$ where $J(x, f)=0$. (See also Section 5 below.)

Finally, (5) is a consequence of a result of Gol'DSHTEn \& Vodop'YANOV [GV, 2.4]. See also [MZ] and [MM] for recent studies on condition (N).

The reader familiar with quasiregular mappings notices that if $f \epsilon$ $W_{\text {loc }}^{1, n}\left(\Omega, \mathbf{R}^{n}\right)$ and if $K(x, f) \in L^{\infty}(\Omega)$, then $f$ is by definition quasiregular, 
and hence either a constant or a discrete and open mapping in each component of $\Omega$, by a fundamental result of RESHETNYAK [R, p. 183]. Recall that a continuous mapping is said to be discrete if the inverse image of each point consists of isolated points.

The question we wish to address is:

Problem. Under which conditions is a mapping of finite dilatation discrete and open?

Because this problem is of a local nature, we assume from now on that $\Omega$ is bounded and connected, and that all mappings in question are non-constant.

IWANIEC \& ŠVERÁK proved in [IŠ] that if $n=2, f \in W^{1,2}\left(\Omega, \mathbf{R}^{2}\right)$, and $K(x, f) \in L^{1}(\Omega)$, then $f$ is not only discrete and open but admits a factorization $f=\varphi \circ h$, where $h$ is a homeomorphism and $\varphi$ is a holomorphic function. Their proof uses the powerful existence theorem for the two-dimensional Beltrami equation, not available in space. It was conjectured in [IŠ] that for all $n \geqq 3$ and $f \in W^{1, n}\left(\Omega, \mathbf{R}^{n}\right)$ the condition $K(x, f) \in L^{n-1}(\Omega)$ implies that $f$ is a discrete and open mapping. In our first theorem (Theorem 1.7) we confirm this conjecture for quasi-light mappings which satisfy $K(x, f) \in L^{n-1+\varepsilon}$ for some $\varepsilon>0$.

Definition. A continuous mapping $f: \Omega \rightarrow \mathbf{R}^{n}$ is quasi-light if for each $y \in \mathbf{R}^{n}$ the components of the set $f^{-1}(y)$ are compact.

The term quasi-light is due to VÄIsÄL $\ddot{A}$ [V1]. Notice that $f$ is quasi-light, for instance, if it agrees with a discrete mapping in a neighborhood of $\partial \Omega$, in particular, if $f$ is a homeomorphism near $\partial \Omega$. Thus (modulo $\varepsilon$ ) Theorem 1.7 extends some results of BALL [B2] and ŠvERÁK [Š]. We emphasize that we do not assume that $J(x, f)>0$ a.e. as was done in [B2] and [Š]. It is in fact an interesting problem whether $J(x, f)>0$ a.e. provided $K(x, f)$ is integrable to some power $r<\infty$ (see Section 6 below).

In an attempt to dispense with any a priori topological assumptions on $f$, we have to take a slightly different approach. It seems that for our method to work, the regularity requirement $f \in W^{1, n}\left(\Omega, \mathbf{R}^{n}\right)$ is too weak. If we assume instead that $f \in W^{1, p}\left(\Omega, \mathbf{R}^{n}\right)$ for some $p>n$, then we can always find $r=r(n, p)<\infty$ such that $K(x, f) \in L^{r}(\Omega)$ implies $f$ is discrete and open; in fact, it turns out that for $p \geqq n+1 /(n-2)$, any $r>n-1$ works. A further glance at the method (which is a refinement of REsHETNYAK's idea) reveals that what is essential is not the integrability of $D f$, but the integrability of the adjugate adj $D f$ of $D f$, which we recall satisfies

$$
D f(x) \text { adj } D f(x)=J(x, f) \text { Id }
$$

for almost every $x$. Since the entries of adj $D f$ are polynomials of degree $n-1$ of entries of $D f$, we have by Hölder's inequality that

$$
\text { adj } D f(x) \in L^{p /(n-1)}(\Omega)
$$

whenever $f \in W^{1, p}\left(\Omega, \mathbf{R}^{n}\right)$ and $p \geqq n-1$. 
We consider the following classes of mappings:

$$
\mathscr{A}_{p, q}(\Omega)=\left\{f \in W^{1, p}\left(\Omega, \mathbf{R}^{n}\right): \operatorname{adj} D f \in L^{q}\right\},
$$

where $p \geqq n-1$ and $q \geqq p /(p-1)$. It follows from the identity (1.5) that $J(x, f) \in L^{1}(\Omega)$ if $f \in \mathscr{A}_{p, q}(\Omega)$. The classes $\mathscr{H}_{p, q}(\Omega)$ were introduced by BaLL and they have been studied by several people in nonlinear elasticity; see [B1, B2, Š, M, MTY].

Now we are ready to state the main results of this paper:

1.7. Theorem. Suppose that $f \in \mathscr{A}_{p, q}(\Omega)$ is a continuous, quasi-light mapping of finite dilatation, where $p \geqq n-1$ and $q \geqq p /(p-1)$. If $K(x, f) \in L^{n-1+\varepsilon}(\Omega)$ for some $\varepsilon>0$, then $f$ is discrete and open.

1.8. Corollary. If $f \in W^{1, n}\left(\Omega, \mathbf{R}^{n}\right)$ is a quasi-light mapping of finite dilatation and if $K(x, f) \in L^{n-1+\varepsilon}(\Omega)$ for some $\varepsilon>0$, then $f$ is discrete and open.

1.9. Theorem. Suppose that $f \in \mathscr{L}_{p, q}(\Omega)$ is a continuous mapping of finite dilatation, where $p \geqq n-1$ and $q \geqq p /(p-1)$. If $K(x, f) \in L^{r}(\Omega)$ for some $r>n-1$ such that

$$
r \geqq \frac{q}{n(q-1)-q},
$$

then $f$ is discrete and open.

If $p \geqq n$ in these theorems, we do not need to assume that $f$ is continuous, as this follows from Theorem 1.3. If $n-1 \leqq p<n$ in Theorem 1.9, it suffices to assume that $K(x, f)$ is in $L^{r}(\Omega)$ for some $r \geqq n /(n-p)$, as can be seen from the condition $q \geqq p /(p-1)$. Moreover, since any mapping in $W^{1, p}\left(\Omega, \mathbf{R}^{n}\right)$ belongs to $\mathscr{A}_{p, q}(\Omega)$ for some $q \geqq p /(n-1)$, the requirement for $r$ in terms of $p \geqq n$ reads

$$
r \geqq \frac{p}{(n-1)(p-n)},
$$

which in the borderline case $p=n$ means $r=\infty$. In orther words, starting with mappings in $W^{1, n}\left(\Omega, \mathbf{R}^{n}\right)$ we do not find any integrability assumption on $K(x, f)$ (beyond $K(x, f) \in L^{\infty}$ which is ReshetNYAK's theorem) to guarantee the discreteness and openness of $f$, unless an extra condition on adj $D f$ is imposed. However, if $p>n$, then adj $D f$ automatically satisfies something which gives a finite value of $r$. Since

$$
\frac{p}{(n-1)(p-n)}=n-1
$$

for

$$
p=n+\frac{1}{(n-2)} \leqq \frac{4 n}{3}, \quad n>2 .
$$

Theorem 1.9 permits us to deduce the following result: 
1.10. Corollary. Suppose that $n \geqq 3$ and that $f \in W^{1, p}\left(\Omega, \mathbf{R}^{n}\right)$ for some $p \geqq n+1 /(n-2)$. If $K(x, f) \in L^{r}(\Omega)$ for some $r>n-1$, then $f$ is discrete and open.

The example in [B2, p. 317-318] shows that the integrability condition on $K(\cdot, f)$ in both Theorems 1.7 and 1.9 cannot be improved much. Indeed, BaLL exhibits a mapping $f$ in the Sobolev space $W^{1, \infty}\left(\Omega, \mathbf{R}^{n}\right)$ such that $f$ is the identity on the boundary of $\Omega$ and $K(x, f) \in L^{r}(\Omega)$ for all $r<n-1$, yet $f$ takes a line segment to a point. Thus both of our results are quite sharp for mappings with sufficient regularity.

The borderline case $f \in W^{1, n}\left(\Omega, \mathbf{R}^{n}\right)$ remains somewhat problematic, and in light of the above discussion we reformulate the conjecture advanced by IWANIEC \& ŠvERÁK: Let $a(n)$ denote the infimum of exponents $p \geqq n$ so that each Sobolev mapping $f \in W^{1, p}\left(\Omega, \mathbf{R}^{n}\right)$ whose dilatation belongs to $L^{r}(\Omega)$ for some $r>n-1$ is discrete and open. Corollary 1.10 states that $a(n) \leqq n+1 /(n-2) \leqq 4 n / 3$ for $n \geqq 3$ and by [IŠ], $a(2)=2$. Hence we are led to the conjecture $a(n)=n$ for all $n \geqq 2$. This conjecture is slightly weaker than that in [IŠ]. Indeed, for $n=2$ we only need $K(x, f) \in L^{1}(\Omega)$.

\section{The Degenerate Equation Associated with $f$}

Throughout this section we assume that $f \in W^{1, p}\left(\Omega, \mathbf{R}^{n}\right), p \geqq n-1$, is a continuous mapping with dilatation $K=K(\cdot, f)$. By Theorem 1.3 the continuity assumption is superfluous provided $p \geqq n$. We also recall that $\Omega$ is assumed to be a bounded domain and $f$ non-constant.

Define $\mathscr{L}: \Omega \times \mathbf{R}^{n} \rightarrow \mathbf{R}^{n}$ by

where

$$
\mathscr{H}(x, \xi)=\langle G(x) \xi, \xi\rangle^{\frac{n-2}{2}} G(x) \xi,
$$

$$
G(x)=J_{f}(x)^{2 / n}[D f(x)]^{-1}[D f(x)]^{-1^{T}}
$$

if $x \in \Omega$ and $J_{f}(x) \neq 0$ exists, and $G(x)=$ Id otherwise; here $T$ denotes transpose. We also write

$$
F(x, \xi)=\langle G(x) \xi, \xi\rangle^{n / 2} .
$$

Then a calculation (see, e.g., [HKM, p. 269]) shows

$$
\begin{gathered}
F(x, \xi)=\mathscr{L}(x, \xi) \cdot \xi \geqq K(x)^{-1}|\xi|^{n} \quad \text { for almost every } x, \\
|\mathscr{A}(x, \xi)| \leqq K(x)^{n-1}|\xi|^{n-1} \quad \text { for almost every } x, \\
F(x, \xi) \leqq|\xi|^{n} K(x)^{n-1} \quad \text { for almost every } x .
\end{gathered}
$$

Next, let $h(x)=-\log |f(x)|$. Then we have that $h \in W_{\text {loc }}^{1, p}\left(\Omega \backslash Z_{f}\right)$ and

$$
\nabla h(x)=\frac{-D f(x)^{T} f(x)}{|f(x)|^{2}},
$$


where $Z_{f}=f^{-1}(0)$ is the zero-set of $f$. Further,

$$
\begin{aligned}
& \mathscr{H}(x, \nabla h)=\frac{-\operatorname{adj} D f(x) \cdot f(x)}{|f(x)|^{n}} \in L_{\mathrm{loc}}^{q}\left(\Omega \backslash Z_{f}\right), \\
& \mathscr{H}(x, \nabla h) \cdot \nabla h=J(x, f)|f(x)|^{2-n} \in L_{\mathrm{loc}}^{1}\left(\Omega \backslash Z_{f}\right)
\end{aligned}
$$

if $f \in \mathscr{A}_{p, q}(\Omega)$. Moreover, the above integrability conditions hold in any subset of $\Omega$ with positive distance from $Z_{f}$. It is important to observe that we have used the fact that $f$ is of finite dilatation in an essential way in formulas (2.4) and (2.5), namely, if $J(x, f)=0$, then $\mathscr{A}(x, \nabla h)=|\nabla h|^{n-2} \nabla h$, but then because $D f(x)=0$, we also find that $\nabla h(x)=0$ so that (2.4) and (2.5) always hold.

Next we invoke the important identity [BI, p. 271]:

2.6. Proposition. $\mathscr{L}(x, \nabla h)$ is a divergence-free vector field in $\Omega \backslash Z_{f}$; that is,

$$
\int \mathscr{A}(x, \nabla h) \cdot \nabla \phi d x=0
$$

for each $\phi \in C_{0}^{\infty}\left(\Omega \backslash Z_{f}\right)$.

Moreover, if $f \in \mathscr{A}_{p, q}(\Omega)$, then by approximation we see that

$$
\int \mathscr{A}(x, \nabla h) \cdot \nabla v d x=0
$$

for each $v \in W_{0}^{1, s}\left(\Omega \backslash Z_{f}\right), s \geqq q /(q-1)$, provided the support of $\nabla v$ has positive distance to $Z_{f}$.

Equation (2.7) means that $h(x)=-\log |f(x)|$ is a weak solution to the second-order partial differential equation $-\operatorname{div} \mathscr{H}(x, \nabla u)=0$ outside $Z_{f}$. This equation is quasilinear elliptic with bounded coefficients if and only if $f$ is a quasiregular mapping, and in the general case the difficulties arise from the degeneracy described in (2.1) and (2.2). In the bounded-coefficient case there is a well-understood connection between polar sets (i.e., sets where a supersolution can take the value at infinity) and sets of zero capacity, and the latter concept provides a way to metric estimates; see [HKM, Chapters 2 and 7]. We shall mimic this approach as much as possible, and for that purpose we next introduce weighted capacities.

Let $1<s \leqq n$ and let $\omega$ be a nonnegative locally integrable function in $\Omega$. For a compact set $C \subset \Omega$, define

$$
\operatorname{cap}_{s, \omega}(C, \Omega)=\inf _{\psi} \int_{\Omega}|\nabla \psi|^{n} \omega d x,
$$

where the infimum is taken over all $\psi \in C_{0}^{\infty}(\Omega)$ with $\psi=1$ in a neighborhood of $C$. If $\omega \equiv 1$, we have the standard variational $s$-capacity $\operatorname{cap}_{s}(C, \Omega)$. By Hölder's inequality,

$$
\int_{\Omega}|\nabla \psi|^{s} d x \leqq\left(\int_{\Omega}|\nabla \psi|^{n} \omega d x\right)^{s / n}\left(\int_{\Omega} \omega^{s /(s-n)} d x\right)^{(n-s) / n}
$$

for $1<s<n$, and hence

$$
\operatorname{cap}_{n, \omega}(C, \Omega)=0 \Rightarrow \operatorname{cap}_{s}(C, \Omega)=0
$$


provided $\omega^{-1} \in L^{s /(n-s)}(\Omega)$. If $\omega$ is bounded, we can take the infimum in (2.9) over continuous functions $\psi \in W_{0}^{1, s}(\Omega)$ without affecting the value of $\operatorname{cap}_{s, \omega}(C, \Omega)$. Finally we recall the well-known Hausdorff dimension estimates:

$$
\operatorname{cap}_{s}(C, \Omega)=0 \Rightarrow \operatorname{dim}_{H} C \leqq n-s
$$

for $1<s \leqq n$. See, e.g., [HKM, 2.24].

\section{Proof of Theorem 1.7}

We first show that $C(y)=f^{-1}(y)$ has Hausdorff dimension less than 1 for each $y \in \mathbf{R}^{n}$; in particular, $C(y)$ is a totally disconnected set for each $y \in \mathbf{R}^{n}$, which means that $f$ is a light mapping. To prove this, we assume, for simplicity, that $y=0$. Denote $C(0)=Z_{f}$. Because $f$ is quasi-light, we may further assume that $Z_{f}$ is a compact and non-empty subset of $\Omega$, and that $f$ is continuous in a neighborhood of $\partial \Omega$ (cf. [V1, Theorem 3.1]).

Consider $h(x)=-\log |f(x)|$. Since $h(x) \rightarrow \infty$ as $x \rightarrow Z_{f}$, we may further assume that

$$
\Omega=\{x: h(x)>0\}
$$

and that $h(x) \rightarrow 0$ as $x \rightarrow \partial \Omega$. For $M>0$ write

$$
E_{M}=\{x \in \Omega: h \geqq M>0\} .
$$

Then $E_{M}$ is compact and $Z_{f} \subset E_{M}$. Pick $\phi \in C_{0}^{\infty}(\Omega)$ such that $\phi=1$ in a fixed neighborhood $U$ of $Z_{f}$. Then $E_{M} \subset U$ for all large $M$, and the support of the gradient of the function

$$
v_{M}=\phi-\min \left\{\frac{h}{M}, 1\right\} \in W_{0}^{1, p}\left(\Omega \backslash Z_{f}\right)
$$

stays away from $Z_{f}$. Because $p \geqq q /(q-1)$ by assumption, (2.8) yields

$$
0=\int_{\Omega \backslash E_{M}} \mathscr{L}(x, \nabla h) \cdot \nabla v_{M} d x=\int_{\Omega \backslash E_{M}} \mathscr{L}(x, \nabla h) \cdot\left(\nabla \phi-\frac{\nabla h}{M}\right) d x
$$

and hence (see (2.4) and (2.5))

$$
\begin{aligned}
& M^{n-1} \int_{\Omega \backslash E_{M}} \mathscr{A}\left(x, \frac{\nabla h}{M}\right) \cdot \frac{\nabla h}{M} d x=\int_{\Omega \backslash E_{M}} \mathscr{A}(x, \nabla h) \cdot \frac{\nabla h}{M} d x \\
& =\int_{\Omega \backslash E_{M}} \mathscr{A}(x, \nabla h) \cdot \nabla \phi d x=\int_{\Omega \backslash \vec{U}} \mathscr{A}(x, \nabla h) \cdot \nabla \phi d x=c<\infty .
\end{aligned}
$$


Applying (2.1) we observe that

$$
\begin{aligned}
M^{n-1} \int_{\Omega \backslash E_{M}} \mathscr{A}\left(x, \frac{\nabla h}{M}\right) \cdot \frac{\nabla h}{M} d x & \geqq M^{n-1} \int_{\Omega \backslash E_{M}}\left|\frac{\nabla h}{M}\right|^{n} K^{-1} d x \\
& \geqq M^{n-1} \operatorname{cap}_{n, K^{-1}}\left(E_{M}, \Omega\right) \geqq M^{n-1} \operatorname{cap}_{n, K^{-1}}\left(Z_{f}, \Omega\right),
\end{aligned}
$$

where we also used the fact that $K=K(x, f) \geqq 1$. Because $c$ does not depend on $M$, we conclude by letting $M \rightarrow \infty$ that

$$
\operatorname{cap}_{n, K^{-1}}\left(Z_{f}, \Omega\right)=0 .
$$

Since $K \in L^{r}(\Omega)$ for some $r>n-1$, we deduce that the variational $s$-capacity of $Z_{f}$ is zero for $s=n r /(1+r)>n-1$, and hence that the Hausdorff dimension of $Z_{f}$ is strictly less than 1 ; see (2.10) and (2.11).

Next, we invoke a theorem of TrTus \& Young [TY], [Ri, I.5.6], which asserts that every sense-preserving light mapping is discrete and open. Therefore we only need to verify that $f$ is sense-preserving. For $p \geqq n$ this follows from Theorem 1.3, while the case $n-1 \leqq p<n$ is established in Section 5 below. We thus conclude the proof of Theorem 1.7.

Remark. That the integrability degree of $K(x, f)$ is at least some $r>n-1$ in Theorem 1.7 was needed only to ensure that the Hausdorff dimension of the inverse image of each point under $f$ is less than 1 . A glance at the above proof together with (2.11) implies the following result: Let $f \in \mathscr{K}_{p, q}(\Omega)$ be a continuous quasi-light mapping of finite dilatation. If $K(x, f) \in L^{r}(\Omega)$ for some $r>1 /(n-1)$, then for each $y \in \mathbf{R}^{n}$ the Hausdorff dimension of $f^{-1}(y)$ is at most $n /(r+1)<n-1$.

The following simple example shows that this estimate for the Hausdorff dimension of the preimage of a point is essentially sharp. Define $f: B(0,2) \rightarrow$ $B(0,1)$ by $f(x)=x(|x|-1)$ if $1<|x|<2$ and $f(x)=0$ otherwise. Then $f \in W^{1, \infty}(B(0,2))$ and a straightforward calculation shows that $K(x, f) \epsilon$ $L^{r}(B(0,2))$ for all $r<1 /(n-1)$. However, $f^{-1}(0)$ is the closed unit ball.

Notice that the case $r=n-1$ gives $\operatorname{dim}_{H} f^{-1}(y) \leqq 1$, and in particular $f^{-1}(y)$ may not be totally disconnected. Therefore our method cannot fully solve the conjecture of IWANIEC \& Šverák.

\section{Proof of Theorem 1.9}

As in the proof of Theorem 1.7, we only need to show that $Z_{f}=f^{-1}(0)$ has Hausdorff dimension less than 1 . The rest follows by invoking the theorem of Titus \& Young and observing that $f$ is sense-preserving (by Theorem 5.1 if $p<n$ and by Theorem 1.3 if $p \geqq n$ ). 
Fix $x_{0} \in \partial Z_{f} \cap \Omega$ and pick a ball $B=B\left(x_{0}, r\right) \subset \subset \Omega$; such an $x_{0}$ exists because $f$ is non-constant. We shall write $\frac{1}{2} B=B\left(x_{0}, r / 2\right)$.

Fix $n-1<s \leqq n$ such that

$$
\begin{aligned}
& s \geqq \frac{q}{q-1}, \\
& r \geqq \frac{s}{n-s} .
\end{aligned}
$$

This selection is possible by the assumption of the theorem. We also understand that $s=n$ if and only if $q=n /(n-1)$, which requires $K \in L^{\infty}(\Omega)$. This is the well-known case of quasiregular mappings which also are covered by our theorem, and it may be worth pointing out that the ensuing proof does not use the relatively deep, albeit well-known regularity theory of nonlinear elliptic equations.

Here and throughout this section q.e. means quasieverywhere with respect to the variational $s$-capacity meaning "everywhere except on a set of zero $s$ capacity". We say that $u \in W^{1, s}(B)$ is quasicontinuous if for each $\varepsilon>0$ there exists an open set $E$ of $s$-capacity less than $\varepsilon$ such that the restriction of $u$ to $B \backslash E$ is continuous. We remind the reader that each function in $W^{1, s}(B)$ has a quasicontinuous representative, and while discussing Sobolev functions we always assume that a quasicontinuous representative has been selected. See [HKM, Chapter 4] or [Z, Chapter 3].

Our strategy is to construct a quasicontinuous function $u \in W_{0}^{1, s}(B)$ such that $u=1$ q.e. on $Z_{f} \cap \frac{1}{2} B$ and that $u=0$ q.e. in $\frac{1}{2} B \backslash Z_{f}$. Because $f$ is continuous and because $x_{0} \in \partial Z_{f} \cap \Omega$, this clearly implies that cap $Z_{f}=0$ and hence, by (2.11), that the Hausdorff dimension of $Z_{f}$ is less than 1 .

We begin by introducing the function space

$$
H=\left\{u \in W_{0}^{1,1}(B): \int_{B} F(x, \nabla u) d x<\infty\right\}
$$

and putting

$$
\|u\|_{F, s}=\left(\int_{B}|u|^{s} d x\right)^{1 / s}+\left(\int_{B} F(x, \nabla u) d x\right)^{1 / n}
$$

Here, as in Section 2,

$$
F(x, \xi)=\langle G(x) \xi, \xi\rangle^{n / 2}=|\Theta(x) \xi|^{n},
$$

$G(x)=\Theta(x)^{T} \Theta(x)$, where $\Theta(x)=J_{f}(x)^{1 / n}[D f(x)]^{-1^{T}}$ if $J_{f}(x) \neq 0$ exists and $\Theta(x)=$ Id otherwise. Now $F(x, \xi)$ is strictly convex and homogeneous of degree $n$ in $\xi$, and we recall from (2.1) and (2.3) that

$$
|\xi|^{n} K(x)^{-1} \leqq F(x, \xi) \leqq|\xi|^{n} K(x)^{n-1}
$$


for almost every $x$, where $K(x)=K(x, f)$. In particular, $F(x, \nabla \psi) \in L^{1}(B)$ for all $\psi \in C_{0}^{\infty}(B)$; moreover, for all $u \in H$,

$$
\begin{aligned}
\int_{B}|\nabla u|^{s} d x & =\int_{B}|\nabla u|^{s} K^{-s / n} K^{s / n} d x \\
& \leqq\left(\int_{B}|\nabla u|^{n} K^{-1} d x\right)^{s / n}\left(\int_{B} K^{s /(n-s)} d x\right)^{(n-s) / n} \\
& \leqq\left.\|K\|_{s}^{s / n}\right|_{(n-s)}\left(\int_{B} F(x, \nabla u) d x\right)^{s / n}
\end{aligned}
$$

Consequently, since

$$
K \in L^{s /(n-s)}(\Omega)
$$

by (4.2), we have the embedding $H \leftrightarrow W_{0}^{1, s}(B)$.

More precisely:

4.4. Lemma. The space $H$ is a reflexive Banach space under the norm $\|\cdot\|_{F, s}$, continuously embedded in $W_{0}^{1, s}(B)$.

Proof. Since

$$
\|u\|_{F, s}=\|u\|_{s}+\left(\int_{B}|\Theta \nabla u|^{n} d x\right)^{1 / n},
$$

it is clear that $\|\cdot\|_{F, s}$ defines a norm.

To show that $H$ is complete, let $\left(u_{j}\right)$ be a Cauchy sequence in $H$. Then, as we noticed above, $\left(u_{j}\right)$ is a Cauchy sequence in $W_{0}^{1, s}(B)$, and thus

in $W_{0}^{1, s}(B)$. Also

$$
u_{j} \rightarrow u \in W_{0}^{1, s}(B)
$$

$$
\int_{B}\left|\Theta \nabla u_{j}-\Theta \nabla u_{i}\right|^{n} d x=\int_{B} F\left(x, \nabla u_{j}-\nabla u_{i}\right) d x
$$

and so $\left(\Theta \nabla u_{j}\right)$ is a Cauchy sequence in $L^{n}\left(B ; \mathbf{R}^{n}\right)$. Let $v \in L^{n}\left(B ; \mathbf{R}^{n}\right)$ satisfy

$$
\Theta \nabla u_{j} \rightarrow v \quad \text { in } L^{n}\left(B ; \mathbf{R}^{n}\right)
$$

and write $w=\Theta^{-1} v$; notice that $\Theta(x)^{-1}$ exists for all $x \in B$ by the definition of $\Theta(x)$. Now

$$
\int_{B} F(x, w) d x=\int_{B}|\Theta w|^{n} d x=\int_{B}|v|^{n} d x<\infty .
$$

Moreover,

$$
\begin{aligned}
\int_{B} F\left(x, \nabla u_{j}-w\right) d x & =\int_{B}\left|\Theta\left(\nabla u_{j}-w\right)\right|^{n} d x \\
& =\int_{B}\left|\Theta \nabla u_{j}-v\right|^{n} d x \rightarrow 0
\end{aligned}
$$

as $j \rightarrow \infty$. In particular, we conclude from the embedding $H \hookrightarrow W_{0}^{1, s}(B)$ that

$$
\nabla u_{j} \rightarrow w \quad \text { in } L^{s}\left(B, \mathbf{R}^{n}\right)
$$


Thus $w=\nabla u, u \in W_{0}^{1, s}(B), \int_{B} F(x, \nabla u) d x<\infty$ by (4.5), and $\left(u_{j}\right)$ converges to $u$ in $H$. This shows that $H$ is complete.

Finally, the norm $\|\cdot\|_{F, s}$ is clearly uniformly convex, whence $H$ is reflexive; see, e.g., [HS, p. 224-225]. The lemma follows.

For a positive integer $k$ let

and set

$$
E_{k}=\{h \geqq k\} \cap \frac{1}{2} \bar{B},
$$

$$
I_{k}=\inf _{\mathscr{G}} \int_{B} F(x, \nabla u) d x \text {, }
$$

where

$$
\mathscr{F} k=\left\{u \in H: u \geqq 1 \text { q.e. on } E_{k}\right\} \text {. }
$$

By (4.3), the family $\mathscr{F}_{k}$ is non-empty. We next establish the existence of a unique minimizer in $\mathscr{F}_{k}$. This follows from the fact that $H$ is reflexive; for completeness we provide the standard details.

Pick a sequence $\left(u_{j}\right)$ of functions in $\mathscr{F}_{k}$ for which

$$
I_{k}=\lim _{j \rightarrow \infty} \int_{B} F\left(x, \nabla u_{j}\right) d x .
$$

Since $F(x, 0)=0$ for almost every $x$, we may also assume that $0 \leqq u_{j} \leqq 1$ q.e. in $B$. Then $\left(u_{j}\right)$ is a bounded sequence in the reflexive Banach space $H$, and so passing to a subsequence if necessary we find that

$$
u_{j} \rightarrow v \in H \text {. }
$$

By the weak lower semicontinuity of norms, we obtain

$$
I_{k}=\liminf _{j \rightarrow \infty} \int_{B} F\left(x, \nabla u_{j}\right) d x \geqq \int_{B} F(x, \nabla v) d x .
$$

Mazur's lemma guarantees that a sequence of convex combinations $\left(v_{j}\right)$ of $u_{j}$ converges to $v$ in $\|\cdot\|_{F, s}$ and by the embedding $H \hookrightarrow W_{0}^{1, s}(B)$ we deduce that $v \in W_{0}^{1, s}(B)$; moreover, $v_{j} \rightarrow v$ in $W_{0}^{1, s}(B)$.

Passing to a subsequence if necessary we may assume that the sequence $\left(v_{j}\right)$ converges to $v$ pointwise q.e. in $B$ (see [HKM, Theorem 4.3]), and hence $v=1$ q.e. on $E_{k}$. In consequence, $v \in \mathscr{F}_{k}$, and we rename it as $u_{k}$. We thus have

$$
I_{k}=\int_{B} F\left(x, \nabla u_{k}\right) d x .
$$

where $u_{k} \in W_{0}^{1, s}(B)$ satisfies $u_{k}=1$ q.e. on $E_{k}$.

For each $k=1,2, \ldots$ we have now obtained a minimizer $u_{k}$ as in (4.6). By using the strict convexity of $F$, we easily obtain the uniqueness:

$$
\int_{B} F\left(x, \nabla u_{k}\right) d x<\int_{B} F(x, \nabla v) d x
$$

for all $v \in \mathscr{F}_{k}$ different from $u_{k}$.

As earlier, write $h(x)=-\log |f(x)|$.

4.8. Lemma. $u_{k} \leqq h / k$ q.e. in $B \backslash E_{k}$ for each $k \geqq 1$. 
Proof. Write $v=k u_{k}$ and put $U=\{v>h\}$. Recall that $v$ is assumed to be quasicontinuous so that $U$ is a measurable set which is well-defined up to zero $s$-capacity. We may assume that $h>0$ on $\partial B$. Since $v=0$ on $\partial B$ and since $0 \leqq v \leqq k$, we conclude that $U \subset B \backslash E_{k}$. In particular, $U \subset \subset \Omega \backslash Z_{f}$. Define

$$
w=\min \{v, h\} \text {. }
$$

Then $w \in W_{0}^{1, s}(B)$, because

$$
F(x, \nabla h)=\frac{J_{f}(x)}{|f(x)|^{n-2}}
$$

by (2.5). In fact, $w=h$ q.e. on $U, w=v$ q.e. on $B \backslash U$, and

$$
\int_{B} F(x, \nabla w) d x=\int_{U} F(x, \nabla h) d x+\int_{B \backslash U} F(x, \nabla v) d x<\infty .
$$

Thus

$$
\int_{B} F(x, \nabla v) d x<\int_{B} F(x, \nabla w) d x
$$

by homogeneity and the uniqueness (4.7), provided $|U|>0$.

If $|U|=0$, there is nothing to prove because $h$ is continuous and $v$ is quasicontinuous (see [HKM, Theorem 4.12]). Thus suppose that $|U|>0$. Then

$$
\begin{aligned}
\int_{B} F(x, \nabla w) d x & =\int_{B \backslash U} F(x, \nabla v) d x+\int_{U} F(x, \nabla h) d x \\
& >\int_{B \backslash U} F(x, \nabla v) d x+\int_{U} F(x, \nabla v) d x
\end{aligned}
$$

by (4.9), and hence

$$
\int_{U} F(x, \nabla v) d x<\int_{U} F(x, \nabla h) d x .
$$

On the other hand,

$$
\begin{aligned}
\int_{U} F(x, \nabla v)-F(x, \nabla h) d x & \geqq \int_{U} \nabla_{\xi} F(x, \nabla h) \cdot(\nabla v-\nabla h) d x \\
& =n \int_{B} \mathscr{A}(x, \nabla h) \cdot(\nabla v-\nabla h) d x
\end{aligned}
$$

by convexity (see [HKM, p. 98]). Next, notice that

$$
w^{\prime}=\max \{v-h, 0\} \in W_{0}^{1, s}\left(B \backslash E_{k}\right) ;
$$

indeed, $w^{\prime}$ can be extended to a quasicontinuous $W^{1, s}$-function in $\mathbf{R}^{n}$ by setting $w^{\prime}=0$ in $E_{k} \cup\left(\mathbf{R}^{n} \backslash B\right)$, and hence the assertion follows from [HKM, 4.5].

Because $s \geqq q /(q-1)$ by (4.1), it follows that

$$
\int_{B} \mathscr{A}(x, \nabla h) \cdot \nabla w^{\prime} d x=0
$$

by (2.8). From the definition of $U$ we conclude that

$$
\int_{U} \mathscr{A}(x, \nabla h) \cdot(\nabla v-\nabla h) d x=0,
$$


and combining this with (4.11) yields

$$
\int_{U} F(x, \nabla h) d x \leqq \int_{U} F(x, \nabla v) d x
$$

which contradicts (4.10). We have thus proved that $|U|=0$, and the lemma follows.

We have constructed a sequence $\left(u_{k}\right)$ of functions in $W_{0}^{1, s}(B)$ such that

$$
\begin{gathered}
u_{k}=1 \quad \text { q.e. on } E_{k}, \\
u_{k} \leqq h / k \quad \text { q.e. on } B \backslash E_{k} .
\end{gathered}
$$

Moreover, this sequence is bounded in $W_{0}^{1, s}(B)$, and hence a sequence of its convex combinations converges to some $u_{0} \in W_{0}^{1, s}(B)$. Passing to a subsequence allows us to assume that the convergence occurs pointwise q.e. in $B$ and that $u_{0}$ is quasicontinuous. It follows that $u_{0}=1$ q.e. on $Z_{f} \cap \frac{1}{2} \vec{B}$ and $u_{0}=0$ q.e. on $\frac{1}{2} B \backslash Z_{f}$, as desired.

This completes the proof of Theorem 1.9.

\section{Sense-Preserving Sobolev Mappings}

Suppose that $f$ is a continuous mapping in $W^{1, p}\left(\Omega, \mathbf{R}^{n}\right)$, where $\Omega$ is an arbitrary open subset of $\mathbf{R}^{n}$. For simplicity, we assume that $f$ is not constant in any of the components of $\Omega$.

If $p \geqq n$, relatively mild analytic conditions guarantee that $f$ is a sensepreserving mapping; it suffices, for instance, to assume that $f$ is of finite dilatation (see Theorem 1.3). For $p<n$ no simple criteria seem to be available, although it would be desirable to have some. In this section we provide a condition under which a mapping $f \in W^{1, p}\left(\Omega, \mathbf{R}^{n}\right)$ of finite dilatation is sensepreserving. The considerations in [Š] most likely would yield more general results in this direction, but we prefer a more elementary argument sufficient to the purposes of this paper.

5.1. Theorem. Suppose that $f \in \mathscr{A}_{p, q}(\Omega)$ is a continuous mapping of finite dilatation, where $p \geqq n-1$ and $q \geqq p /(p-1)$. If the 1-dimensional Hausdorff measure of $f^{-1}(y)$ is zero for each $y \in \mathbf{R}^{n}$, then $f$ is sense-preserving.

Proof. Fix a domain $D \subset C \Omega$ and $y \in f(D) \backslash f(\partial D)$. We must show that $\mu(y, f, D)>0$. Let $E=f^{-1}(y) \cap D$; then $E$ is compact and has zero 1-dimensional Hausdorff measure. In particular, projections of $E$ into the coordinate axes all have zero 1-dimensional Hausdorff measure, and a simple argument then shows that $E$ can be covered by a finite number of open $n$-cubes $Q_{1}, \ldots, Q_{N}$ in $D$ with pairwise disjoint closures such that $\partial Q_{i} \cap E=\emptyset$ for all $i=1, \ldots, N$. (See [G, Lemma 1] for a more precise argument.) Next, by precomposing $f$ with an appropriate bi-Lipschitz mapping we can assume without loss of generality that $E$ is covered by open balls $B_{1}, \ldots, B_{N}$ whose closures are pairwise disjoint and lie in $D$ such that $\partial B_{i} \cap E=\emptyset$ for all 
$i=1, \ldots, N$. Moreover, by invoking [Š, Proposition 1 and Theorem 3] we can further assume that, for all $i=1, \ldots, N$,

$$
\int_{B_{i}} u(f(x)) J(x, f) d x=\mu\left(y, f, B_{i}\right)
$$

whenever $u$ is a smooth function, supported in the $y$-component of $\mathbf{R}^{n} \backslash f\left(\partial B_{i}\right)$ with $\int u d y=1$. Here we need the assumption that $f \in \mathscr{A}_{p, q}(\Omega)$.

Since $E \subset \cup_{i} B_{i}$ and since the balls are disjoint, by well-known properties of the topological index (see [Ri, I 4.4] or [R, p. 88]) we have that

$$
\mu(y, f, D)=\sum_{i=1}^{N} \mu\left(y, f, B_{i}\right) .
$$

Consequently, it suffices to show that, for all $i=1, \ldots, N$,

$$
\int_{B_{i}} u(f(x)) J(x, f) d x>0
$$

for some $u$ as in (5.2).

To this end, fix $i$ and consider an open ball $B_{\varepsilon}=B(y, \varepsilon)$, where $\varepsilon>0$ is so small that $\bar{B}_{\varepsilon}$ belongs to the $y$-component of $\mathbf{R}^{n} \backslash f\left(\partial B_{i}\right)$. Let $D_{\varepsilon}$ be a component of the open set $f^{-1}\left(B_{\varepsilon}\right) \cap B_{i}$ containing points from $E$ (we may naturally assume that $\left.B_{i} \cap E \neq \emptyset\right)$. It clearly suffices to show that the measure of $\left\{z \in D_{\varepsilon}: J(z, f)>0\right\}$ is positive. Suppose on the contrary that $J(z, f)=0$ a.e. in $D_{\varepsilon}$. Then $D f=0$ a.e. in $D_{\varepsilon}$ since $f$ has finite dilatation, and the absolute continuity on lines of Sobolev functions (see [Z, p. 44]) now implies that $f$ is constant in $D_{\varepsilon}$; more precisely, $f\left(D_{\varepsilon}\right)=\{y\}$. An easy argument using the continuity of $f$ then shows that $D_{\varepsilon}$ must coincide with $B_{i}$, but this is a contradiction since $y \in \mathbf{R}^{n} \backslash f\left(\partial B_{i}\right)$. The theorem follows.

Remarks. (a) For the above proof it is essential that $f^{-1}(y)$ has zero 1-dimensional Hausdorff measure. In fact, the covering argument may fail if we only assume that $f^{-1}(y)$ is totally disconnected, as can be seen by considering the well-known Antoine's necklace (a compact, totally disconnected set in $\mathbf{R}^{3}$ whose complement is not simply-connected).

(b) The techniques in [R, p. 173] and [MZ] can be combined to obtain further conditions which guarantee that a Sobolev mapping $f \in W^{1, p}\left(\Omega, \mathbf{R}^{n}\right)$ is sense-preserving for $p<n$. For instance, consider the following Jacobian approximation property: $J(x, f) \in L^{1}(\Omega)$ and there exists a sequence of smooth mappings $f_{i}: \Omega \rightarrow \mathbf{R}^{n}$ such that $f_{i} \rightarrow f$ locally uniformly in $\Omega$ and $J\left(x, f_{i}\right) \rightarrow$ $J(x, f)$ locally in $L^{1}(\Omega)$.

One can show that if $p \geqq 1$ and if $f \in W^{1, p}\left(\Omega, \mathbf{R}^{n}\right)$ is of finite dilatation and has the Jacobian approximation property, then $f$ is sense-preserving. It would be interesting to find some analytic conditions on $f$ which are weaker than being in $W^{1, n}\left(\Omega, \mathbf{R}^{n}\right)$ and which would guarantee the Jacobian approximation property. 


\section{Homeomorphisms with Integrable Dilatation}

In this section we assume that $f$ is a mapping in $W^{1, n}\left(\Omega, \mathbf{R}^{n}\right)$ with finite dilatation $K(\cdot, f)$. For simplicity, we further assume that $\Omega$ is bounded and connected. Then Theorem 1.3 implies that $f$ is continuous and satisfies condition $(\mathrm{N})$, that is, $f$ maps sets of measure zero into sets of measure zero.

Definition. We say that a continuous mapping $f: \Omega \rightarrow \mathbf{R}^{n}$ satisfies condition $\left(\mathrm{N}^{-1}\right)$ if $\left|f^{-1}(E)\right|=0$ whenever $|E|=0$ for a measurable set $E \subset \mathbf{R}^{n}$.

It is not difficult to give examples of Lipschitz homeomorphisms of finite dilatation which do not satisfy condition $\left(\mathrm{N}^{-1}\right)$. However, quasiregular mappings satisfy $\left(\mathrm{N}^{-1}\right)$ and therefore it is natural to ask whether some integrability condition on $K(\cdot, f)$ implies the same conclusion, at least when $f$ satisfies certain natural topological assumptions such as discreteness and openness. In the quasiregular case this is usually done by first establishing that the branch set $B_{f}$ of $f$ has zero measure and then exploiting the fact that the inverse of a quasiconformal mapping (i.e., a homeomorphic quasiregular mapping) is also quasiconformal. Recall that the branch set $B_{f}$ is the set of all points where $f$ does not define a local homeomorphism.

We have the following result:

6.1. Theorem. Suppose that $f \in W^{1, n}\left(\Omega, \mathbf{R}^{n}\right)$ is a homeomorphism with dilatation $K(x, f) \in L^{n-1}(\Omega)$. Then $f^{-1}$ belongs to $W^{1, n}(f(\Omega))$ and is of finite dilatation with $K\left(y, f^{-1}\right)=K\left(f^{-1}(y), f\right)^{n-1}$ for almost every $y \in f(\Omega)$. In particular, $J(x, f)>0$ for almost every $x \in \Omega$ and $f$ satisfies condition $\left(\mathrm{N}^{-1}\right)$.

From this result one easily deduces:

6.2. Corollary. If $f \in W^{1, n}\left(\Omega, \mathbf{R}^{n}\right)$ satisfies $K(x, f) \in L^{n-1}(\Omega)$, then $\left|B_{f}\right|=0$ if and only if $J(x, f)>0$ for almost every $x \in \Omega$.

We do not know whether $\left|B_{f}\right|=0$ even if it is assumed that $f \in$ $W^{1, n}\left(\Omega, \mathbf{R}^{n}\right)$ is discrete and open and $K(x, f) \in L^{r}(\Omega)$ for some $r<\infty$.

Proof. To prove the first assertion we use the reasoning in [BI, Theorem 9.1, p. 314]. In fact, we employ the change of variables formula:

$$
\int_{\Omega} u(f(x)) J(x, f) d x=\int_{f(\Omega)} u(y) d y
$$

for all nonnegative measurable $u$ in $f(\Omega)$ such that the integrals converge. This follows, e.g., from [V2, Theorem 24.8] by standard arguments; note that we need both the fact that $f$ satisfies condition $(\mathrm{N})$ and that $J(x, f)=0$ for almost every $x$ in any set $E \subset \Omega$ such that $|f(E)|=0$ (the latter fact follows from [MZ, Theorem 3.1]). Now with formula (6.3) in hand, we can apply the argument in [BI, pp. 314, 315] verbatim to conclude

$$
\int_{f(\Omega)} D \varphi(y)^{T} g(y) d y=-\int_{\Omega} \operatorname{adj} D f(x)^{T} \varphi(f(x)) d x
$$


for any mapping $\varphi \in C_{0}^{\infty}\left(f(\Omega), \mathbf{R}^{n}\right)$, where $g=f^{-1}$. By basic linear algebra, we have the inequality

$$
|\operatorname{adj} D f(x)|=\left|D f(x)^{-1} J(x, f)\right| \leqq K(x, f)^{(n-1) / n} J(x, f)^{(n-1) / n}
$$

for almost every $x$ (see, e.g., [R, p. 59]); note that here again we use the fact that $f$ is of finite dilatation, which implies that $D f(x)=\operatorname{adj} D f(x)=0$ for almost every $x$ such that $J(x, f)=0$. Thus by (6.4) and (6.3)

$$
\begin{aligned}
\left|\int_{f(\Omega)} D \varphi(y)^{T} g(y) d y\right| & \leqq \\
\Omega & K(x, f)^{(n-1) / n} J(x, f)^{(n-1) / n}|\varphi(f(x))| d x \\
& \leqq\left(\int_{\Omega} K(x, f)^{n-1} d x\right)^{1 / n}\left(\int_{\Omega}|\varphi(f(x))|^{n /(n-1)} J(x, f) d x\right)^{(n-1) / n} \\
& \leqq\left(\int_{\Omega} K(x, f)^{n-1} d x\right)^{1 / n}\left(\int_{f(\Omega)}|\varphi(y)|^{n /(n-1)} d y\right)^{(n-1) / n}
\end{aligned}
$$

for all mappings $\varphi \in C_{0}^{\infty}\left(f(\Omega), \mathbf{R}^{n}\right)$. This implies (see [BI, (1.23)]) that $g=f^{-1} \in W^{1, n}\left(f(\Omega), \mathbf{R}^{n}\right)$ with

$$
\int_{f(\Omega)}|D g(y)|^{n} d y \leqq \int_{\Omega} K(x, f)^{n-1} d x .
$$

A theorem of REshetnyak [R, Corollary 1, p. 182] asserts that homeomorphisms in $W^{1, n}$ satisfy condition $(\mathrm{N})$. Therefore the change of variables formula holds for $f^{-1}$ as well, and we find

$$
\int_{\Omega} K(x, f)^{n-1} d x=\int_{f(\Omega)} K\left(f^{-1}(y), f\right)^{n-1} J\left(y, f^{-1}\right) d y .
$$

Next, because (6.5) holds true for any subdomain of $\Omega$, we arrive at the pointwise estimate

$$
|D g(y)|^{n} \leqq K\left(f^{-1}(y), f\right)^{n-1} J(y, g)
$$

for almost every $y \in f(\Omega)$, and it remains to observe that $K(y, g)$ is finite a.e. in $f(\Omega)$ because $K(x, f)$ is finite a.e. in $\Omega$ and $f$ satisfies condition $(\mathrm{N})$. This completes the proof of both the first assertion of the theorem and the assertion that $f$ satisfies condition $\left(\mathrm{N}^{-1}\right)$.

Finally, that $J(x, f)>0$ a.e. follows from the change of variables formula (6.3) because $f$ satisfies condition $\left(\mathrm{N}^{-1}\right)$.

Remark. To illustrate the sharpness of Theorem 6.1, consider the mapping $f\left(x_{1}, x_{2}\right)=\left(x_{1}, x_{2}^{\alpha}\right), \alpha>1$, in the unit disk $\mathbf{B}^{2}$ in $\mathbf{R}^{2}$. A calculation shows that $K(x, f) \in L^{r}\left(\mathbf{B}^{2}\right)$ for all $r<1 /(\alpha-1)$, but $f^{-1} \in W^{1,2}\left(\mathbf{B}^{2}\right)$ only if $\alpha<2$.

Note added in proof. Recently J. MANFREDI \& E. VILLAMOR have announced a positive answer to the conjecture stated at the end of the Introduction. 
Acknowledgements. We thank TADEUSz IwANIEC and JUAN MANFREDT for inspiring discussions. HeInONEN is supported by NSF Grant DMS 91-48183 and both HeINONEN and Koskela are supported by the Finnish Academy.

\section{References}

[B1] BaLL, J. M., Convexity conditions and existence theorems in nonlinear elasticity, Arch. Rational Mech. Anal. 63 (1978), 337-403.

[B2] BALL, J. M., Global invertibility of Sobolev functions and the interpenetration of matter, Proc. Royal Soc. Edinburgh 88A (1981), 315-328.

[BI] BoJARSKI, B. \& T. IWANIEC, Analytical foundations of the theory of quasiconformal mappings in $\mathbf{R}^{n}$, Ann. Acad. Sci. Fenn. Ser. A. I. Math. 8 (1983), 257-324.

[G] GEHRING, F. W., The Hausdorff measure of sets which link in Euclidean space, Contributions to Analysis: A Collection of Papers Dedicated to Lipman Bers, Academic Press, New York, 1974.

[GV] Gól'DȘTEIN, V. M. \& S. K. VodoP'YANOV, Quasiconformal mappings and spaces of functions with generalized derivatives, Sibirsk. Mat. Z. 17 (1976), 515-531.

[HKM] Hennonen, J., T. Kilpelärnen \& O. Martio, Nonlinear potential theory of degenerate elliptic equations, Oxford University Press, 1993.

[HS] Hewitt, E. \& K. Stromberg, Real and abstract analysis, Springer-Verlag, Berlin-Heidelberg, 1965.

[IS] : IWANIEC, T. \& C. SBORDONE, On the integrability of the Jacobian under minimal hypotheses, Arch. Rational Mech. Anal. 119 (1992), 129-143.

[IŠ] IWANIEC, T. \& V. ŚVERÁK, On mappings with integrable dilatation, to appear in Proc. Amer. Math. Soc.

[MM] MalÝ, J. \& O. Martio, Lusin's condition (N) and mappings of the class $W^{1, n}$, Preprint, the University of Jyväskylä (1992).

[M] MANFRedi, J. J., Weakly monotone functions, preprint (1993).

[MZ] MARTIO, O. \& W. P. ZIEMER, Lusin's condition (N) and mappings with nonnegative Jacobians, Michigan Math. J. 39 (1992), 495-508.

[MTY] MÜLLER, S., Q. TANG \& B. S. YANG, On a new class of elastic deformations not allowing for cavitation, Ann. l'Inst. H. Poincaré, Analyse non linéaire (to appear).

[R] ReshetNYAK, YU. G., Space mappings with bounded distortion, Translation of Mathematical Monographs 73, American Mathematical Society, Providence, 1989.

[Ri] Rickman, S., Quasiregular mappings, Springer-Verlag, to appear.

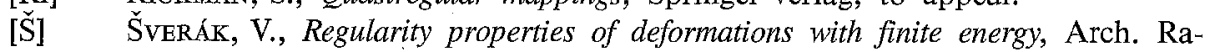
tional Mech. Anal. 100 (1988), 105-127.

[TY] Titus, C. J. \& G. S. Young, The extension of interiority, with some applications, Trans. Amer. Math. Soc. 103 (1962), 329-340.

[V1] V̈̈̈sÄLÄ, J., Minimal mappings in euclidean spaces, Ann. Acad. Sci. Fenn. Ser. A I 366 (1965), 1-22.

[V2] VÄIs⿱̈亡̈̈̈, J., Lectures on n-dimensional quasiconformal mappings, Lecture Notes in Math. 229, Springer-Verlag, Berlin-Heidelberg-New York, 1971.

[Z] ZIEMER, W. P., Weakly Differentiable Functions, Springer-Verlag, New York, 1989.

University of Michigan

Department of Mathematics

Ann Arbor, Michigan 48109 